\title{
Diffusion-based Channel Characterization in Molecular Nanonetworks
}

\author{
Ignacio Llatser*, Eduard Alarcón*, Massimiliano Pierobon ${ }^{\dagger}$ \\ *NaNoNetworking Center in Catalunya (N3Cat) \\ Universitat Politècnica de Catalunya \\ Barcelona, Spain \\ E-mail: 1latser@ac.upc.edu, ealarcon@eel.upc.edu \\ $\dagger$ Broadband Wireless Networking Laboratory \\ School of Electrical and Computer Engineering \\ Georgia Institute of Technology, Atlanta, Georgia 30332, USA \\ E-mail: maxp@ece.gatech.edu
}

\begin{abstract}
Nanotechnology is enabling the development of devices in a scale ranging from one to a few hundred nanometers, known as nanomachines. How these nanomachines will communicate is still an open debate. Molecular communication is a promising paradigm that has been proposed to implement nanonetworks, i.e., the interconnection of nanomachines. Recent studies have attempted to model the physical channel of molecular communication, mainly from a communication or information-theoretical point of view. In this work, we focus on the diffusion-based molecular communication, whose physical channel is governed by Fick's laws of diffusion. We characterize the molecular channel following two complementary approaches: first, we obtain the channel impulse response, transfer function and group delay; second, we propose a pulse-based modulation scheme and we obtain analytical expressions for the most relevant performance evaluation metrics, which we also validate by simulation. Finally, we compare the scalability of these metrics with their equivalents in a wireless electromagnetic channel. We consider that these results provide interesting insights which may serve designers as a guide to implement future molecular nanonetworks.
\end{abstract}

\section{INTRODUCTION}

Nanotechnology, the study of nanometer-scale systems, is a multidisciplinary field with potential applications in the biomedical [1], environmental [2] and industrial fields [3]. A nanomachine is the most basic functional unit able to perform very simple tasks at the nanoscale. These tasks include computing, data storage, sensing and actuation.

Nanonetworks, the interconnection of nanomachines, provide means for cooperation and information sharing among them, allowing nanomachines to fulfill more complex tasks. A sample application from the biomedical field would be an intelligent disease detection and targeted drug delivery system, constituted by an intra-body distributed network of nanosensors and nanoactuators.

Several techniques have been proposed to interconnect nanomachines by drawing inspiration from biology [4], [5]. Among them is calcium signaling [6], one of the most common techniques for intra- and inter-cellular communication, based on the use of calcium ions $\left(\mathrm{Ca}^{2+}\right)$ to encode and transmit information. As other researchers have previously done [7], [8], we propose calcium signaling as a method to realize communication among nanomachines in the short range.

The propagation of a low concentration of calcium ions in a fluid environment is a particular case of molecular diffusion. Several authors have attempted to model the molecular diffusion-based physical channel. Some researchers have focused on the channel transfer function [9], while others have determined the channel capacity from an informationtheoretical point of view [10], [11], [12].

In this work, we focus on a diffusion-based molecular communication whose physical channel is governed by Fick's laws of diffusion. For this channel, we recover the impulse response, the transfer function and the group delay. Further, we propose a pulse-based modulation scheme which we use to obtain analytical expressions of relevant communication metrics, namely, the pulse delay, amplitude and width. These results are validated by means of simulation. Furthermore, the highest achievable bandwidth is analyzed and a scenario with multiple simultaneous transmitters is evaluated.

The remainder of this paper is organized as follows. Section II describes the molecular diffusion-based channel, for which the impulse response is shown, and the proposed modulation scheme. In Section III, we briefly present N3Sim, a simulator for diffusion-based molecular communication. In Section IV, we represent the channel transfer function and the group delay of the molecular channel. In Section V, we obtain analytical expressions of relevant communication metrics in the described scenario, and we validate them with N3Sim. Next, in Section VI we analyze the highest achievable bandwidth and in Section VII we examine the case in which multiple users transmit simultaneously. Finally, Section VIII concludes the paper.

\section{Molecular Diffusion-Based Channel And MOdulation SCHEME}

The molecular channel that we aim to characterize can be described as a set of nanomachines which communicate 
through molecular diffusion in a fluid medium. Transmitter nanomachines encode the information to be sent into a molecular release pattern. The emitted molecules cause a variation in their local concentration, which propagates throughout the medium. Receivers are able to estimate the concentration of molecules in their neighborhood and, from this measurement, recover the release pattern and decode the sent information.

In our envisaged scenario, the concentration of emitted molecules is much lower than the concentration of the fluid molecules. Under these conditions, we assume that interaction among the emitted molecules (e.g., collisions and electrostatic forces) can be neglected. An example of this kind of scenario is calcium signaling among cells, where extracellular concentration of calcium ions is in the millimolar range [13], while the concentration of water (the main component of extracellular fluid) is of 55.5 molar, more than 4 orders of magnitude higher.

In this scenario, each of the molecules released by a transmitter moves according to Brownian motion. Since the movement of each molecule is independent, molecular diffusion can be modeled by Fick's laws of diffusion [14] with a homogeneous diffusion coefficient both in space and time. In this case, the diffusion equations are linear [15].

We propose a pulse-based modulation scheme for diffusionbased molecular communication. According to this scheme, whenever a transmitter nanomachine wants to communicate some information to its neighbors (e.g., after it detects an infectious virus [16]), it instantaneously releases a pulse of molecules. This creates a spike in the molecular concentration at the transmitter location, which then propagates through space and time. The propagation of this pulse can be analytically modeled by solving Fick's laws of diffusion. If the transmitter releases $Q$ molecules at the instant $t=0$, the molecular concentration at any point in space is given by [17]:

$$
c(r, t)=\frac{Q}{(4 \pi D t)^{3 / 2}} e^{-r^{2} / 4 D t}
$$

where $D$ is the diffusion coefficient of the medium, $t$ is time and $r$ is the distance from the transmitter location.

It should be noted that, since the emitted molecules do not interact among them, this scheme allows for simultaneous transmissions by multiple nanomachines. Interferences may be avoided if transmitters use different molecule types, in a mechanism known as Molecular Division Multiple Access (MDMA) [18].

\section{N3Sim, a Simulator for Molecular COMMUNICATION}

In order to validate the analytical results derived in this work, we use the simulation framework N3Sim [19]. This tool allows the molecule-by-molecule simulation of diffusionbased molecular networks, with multiple transmitters and receivers suspended in a 3-dimensional fluid medium. Each transmitter is modeled as a punctual nanomachine with a fixed location. Transmitters encode the information by releasing molecules into the medium with a user-specified pattern. The emitted molecules move according to Brownian motion, as a

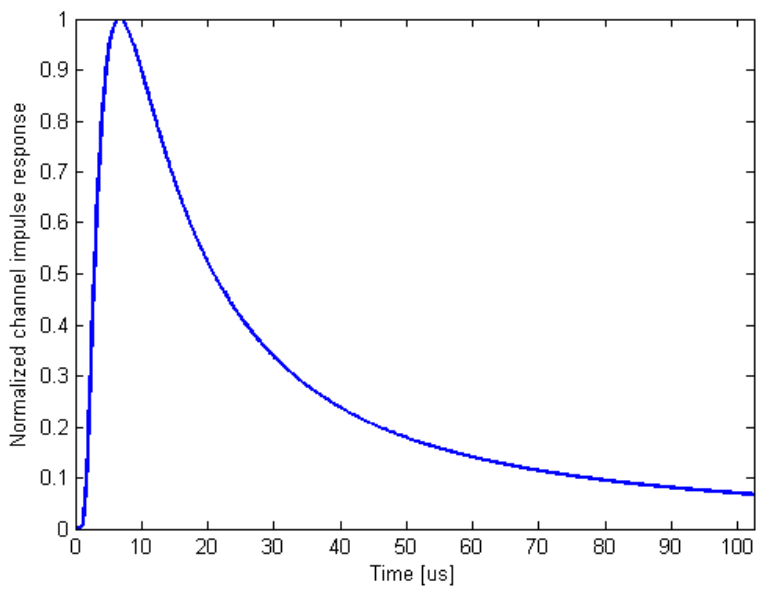

Fig. 1. Normalized channel impulse response.

result of collisions with the smaller fluid molecules. Finally, receivers are modeled as spherical nanomachines which are able to count the number of molecules in a surrounding volume, thus estimating the local concentration. From this measurement, the transmitted information can be decoded.

The main output of N3Sim is the concentration as a function of time measured by each of the receivers. The interested reader can find more details about N3Sim on our website [19].

\section{Molecular Channel Analysis}

Eq. (1) allows to obtain the concentration measured by a receiver located at a distance $r$ from the transmitter as a function of time. Since the previously described molecular channel is linear and time-invariant, this measure can be interpreted as the channel response to an impulse of molecules, i.e., the channel impulse response.

Fig. 1 shows the impulse response of the molecular channel, normalized to values between 0 and 1 . We set the transmission distance to $r=200 \mathrm{~nm}$ and the diffusion coefficient to $D=1 \mathrm{~nm}^{2} / \mathrm{ns}$, similar to the diffusion coefficient of ionic calcium in cytoplasm [20]. We can observe that the concentration initially measured by the receiver is zero, but it sharply increases until reaching its maximum. The time instant at which this maximum occurs can be interpreted as the pulse delay. After the concentration peak is reached, the impulse response slowly decreases, forming a long tail due to the effect of diffusion.

We then obtain the channel transfer function by computing the Fourier transform of the impulse response. The magnitude of the channel transfer function, shown in Fig. 2 in dB, can be interpreted as the channel attenuation. The results indicate that only low-frequency signals can be reliably transmitted through the channel. We observe a notch at $f=500 \mathrm{kHz}$, related to the delay caused by the diffusion process.

Fig. 3 shows the molecular channel group delay. At low frequencies, we observe two peaks: a positive peak at $f=0 \mathrm{~Hz}$ and a negative one at $f=500 \mathrm{kHz}$. The latter one is due to the delay that causes the notch in the channel transfer function at 


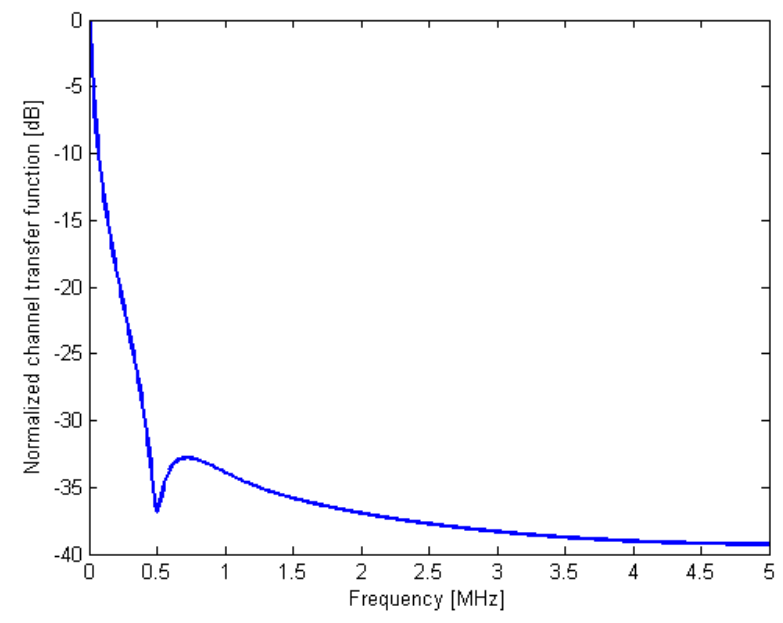

Fig. 2. Magnitude of the normalized channel transfer function in $\mathrm{dB}$.

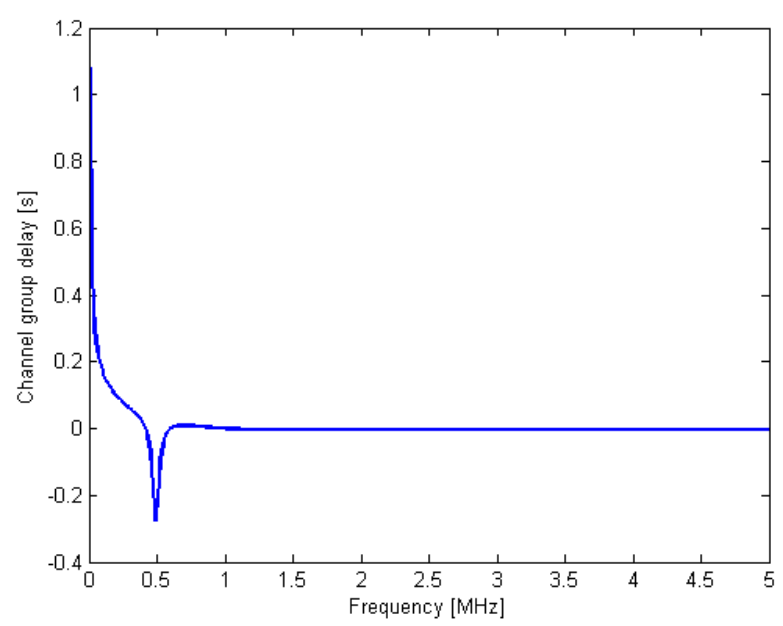

Fig. 3. Channel group delay.

the same frequency. At higher frequencies, the channel group delay is approximately zero.

In Fig. 4 and Fig. 5, we plot the magnitude of the normalized channel transfer function and the group delay, respectively, as a function of both the frequency and the transmission distance. The transmission distance ranges from 100 to $450 \mathrm{~nm}$. As expected, the channel attenuation increases both with the increase in frequency and in transmission distance. The channel group delay also increases with the the distance from the transmitter, and is nearly zero for frequencies higher than a few hundreds of $\mathrm{kHz}$.

\section{Communication Metrics}

Eq. (1) can also be interpreted as the pulse equation, since it shows the evolution of a molecular pulse in space and time. Considering the pulse-based modulation scheme introduced in Section II, an alternative path to explore the characteristics of the molecular channel is to direcly analyze the pulse equation.

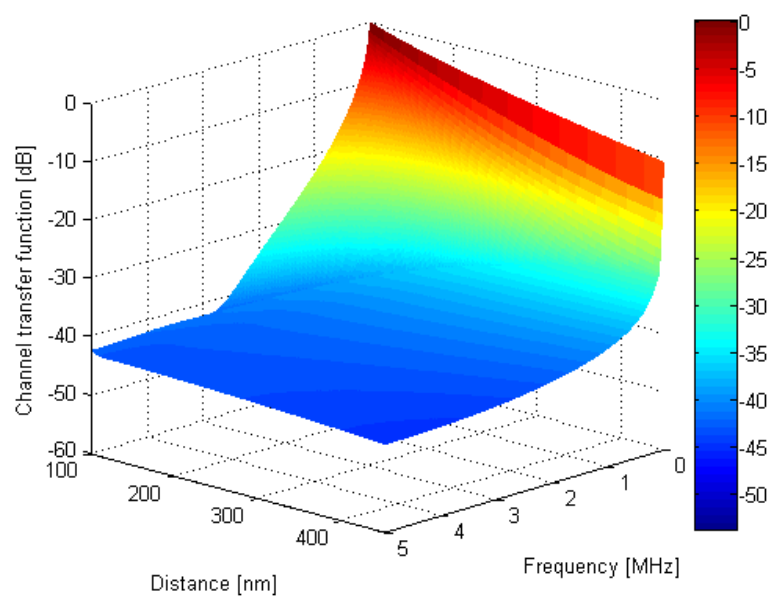

Fig. 4. Magnitude of the normalized channel transfer function in $\mathrm{dB}$ as a function of the transmission distance.

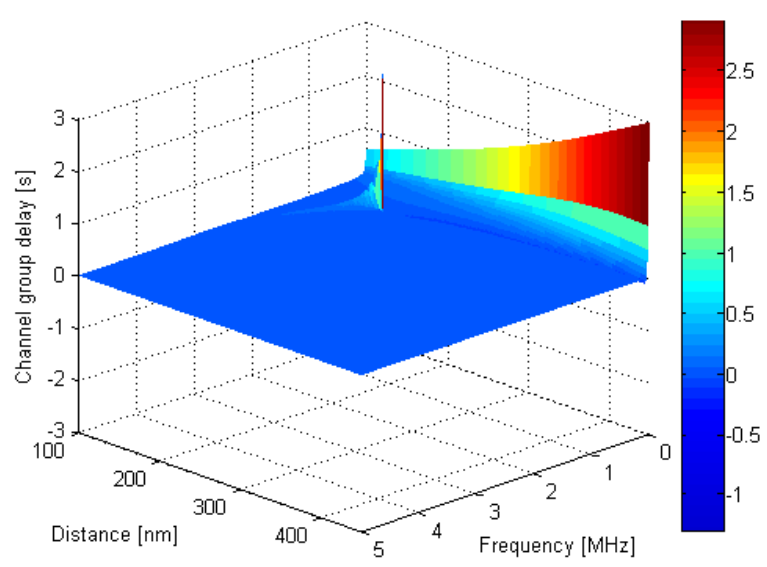

Fig. 5. Channel group delay as a function of the transmission distance.

With this purpose, we consider several metrics that will enable the assessment of the communication performance in this scenario. We focus on three of them: the pulse delay, the pulse amplitude and the pulse width. First, we obtain analytical expressions for these metrics and validate them by simulation. Then, we compare the scalability of these metrics with their equivalent in a wireless electromagnetic channel.

\section{A. Pulse Delay}

In order to find the pulse delay, we compute the time instant for which the pulse equation reaches its global maximum. As we observe in Fig. 1, this function has only one local maximum, which is also its global maximum. We can therefore compute the position of this maximum by taking the time derivative of the function and finding the time instant at which it is equal to zero:

$$
\frac{d c(r, t)}{d t}=\frac{d}{d t} \frac{Q e^{-r^{2} / 4 D t}}{(4 \pi D t)^{3 / 2}}=0
$$




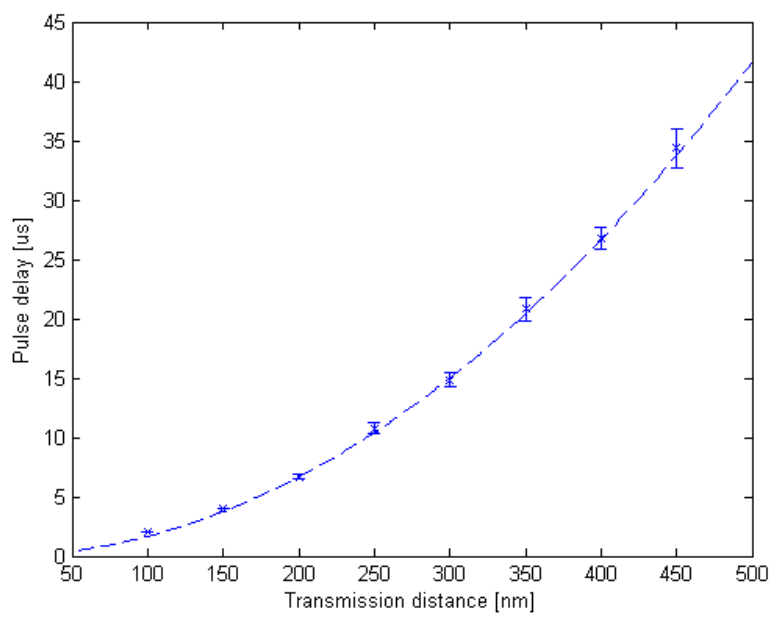

Fig. 6. Plot of the pulse delay as a function of the transmission distance. The dashed line corresponds to the analytical expression, and the crosses show the simulation results with $95 \%$ confidence intervals.

From this equation, by isolating the variable $t$ we can obtain the time at which the pulse has its maximum $t_{d}$. This time can be interpreted as the pulse delay:

$$
t_{d}=\frac{r^{2}}{6 D}
$$

Note that the pulse delay is inversely proportional to the diffusion coefficient $D$. Hence, the higher the diffusion coefficient, the faster molecular pulses will propagate.

In order to validate this result, we simulate the transmission of a $10^{7}$ molecule pulse using N3Sim. The diffusion coefficient is set to $D=1 \mathrm{~nm}^{2} / \mathrm{ns}$ and the local molecular concentration is measured at distances from 100 to $450 \mathrm{~nm}$, at intervals of $50 \mathrm{~nm}$. We will use these same conditions throughout this work, unless otherwise stated. Fig. 6 shows a comparison between the analytical expression of the pulse delay (dashed line) and the averaged results obtained with N3Sim after 30 simulation runs with $95 \%$ confidence intervals.

\section{B. Pulse Amplitude}

It is also worth investigating the variation of the pulse amplitude over space, which may be interpreted as the channel attenuation. We obtain this amplitude by evaluating the pulse equation at the time instant at which the pulse reaches its maximum value, which we have previously found in Eq. (3):

$$
c_{\max }=\left.c(r, t)\right|_{t=t_{d}}=\left(\frac{3}{2 \pi e}\right)^{3 / 2} \frac{Q}{r^{3}}
$$

It is interesting to note that, as opposed to the pulse delay, the pulse amplitude is independent from the diffusion coefficient. In consequence, the diffusion coefficient of the medium will have no effect on the attenuation of the molecular pulses throughout space.

As done in the previous section, we validate this result by means of simulation. Considering a pulse transmission

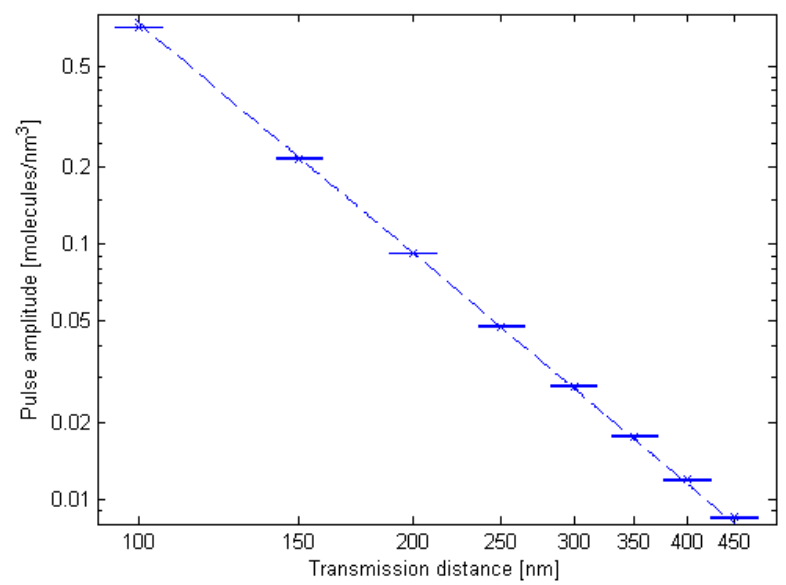

Fig. 7. Log-log plot of the pulse amplitude as a function of the transmission distance. The dashed line corresponds to the analytical expression, and the crosses show the simulation results with $95 \%$ confidence intervals.

under the same conditions used to validate the pulse delay, we measure the pulse amplitude as a function of the transmission distance. Fig. 7 shows a log-log plot that compares the analytical expression (dashed line) with the simulation results with $95 \%$ confidence intervals, which confirms the correctness of Eq. (4).

\section{Pulse Width}

Another important metric is the pulse width, since it will be the main constraint on the achievable bandwidth. As it is usually done in electromagnetic communications, we compute the pulse width at the $50 \%$ level, i.e., the time interval at which the pulse has an amplitude greater than half of its maximum value:

$$
c(r, t)=\frac{Q}{(4 \pi D t)^{3 / 2}} e^{-r^{2} / 4 D t}=\frac{c_{\max }}{2}=\frac{1}{2}\left(\frac{3}{2 \pi e}\right)^{3 / 2} \frac{Q}{r^{3}}
$$

We obtain the following expression by isolating the time variable:

$$
t=-\frac{r^{2}}{6 D W\left(-\frac{1}{2^{2 / 3} e}\right)}
$$

where $W$ is the Lambert $\mathrm{W}$ function [21]. This equation has two solutions, corresponding to the two time instants at which the pulse amplitude is equal to half of its maximum value. These instants are given by:

$$
t_{1}=\frac{0.0728}{D} r^{2} \quad t_{2}=\frac{0.5229}{D} r^{2}
$$

Finally, we can obtain the expression of the pulse width $t_{w}$ by subtracting these two instants:

$$
t_{w}=t_{2}-t_{1}=\frac{0.4501}{D} r^{2}
$$




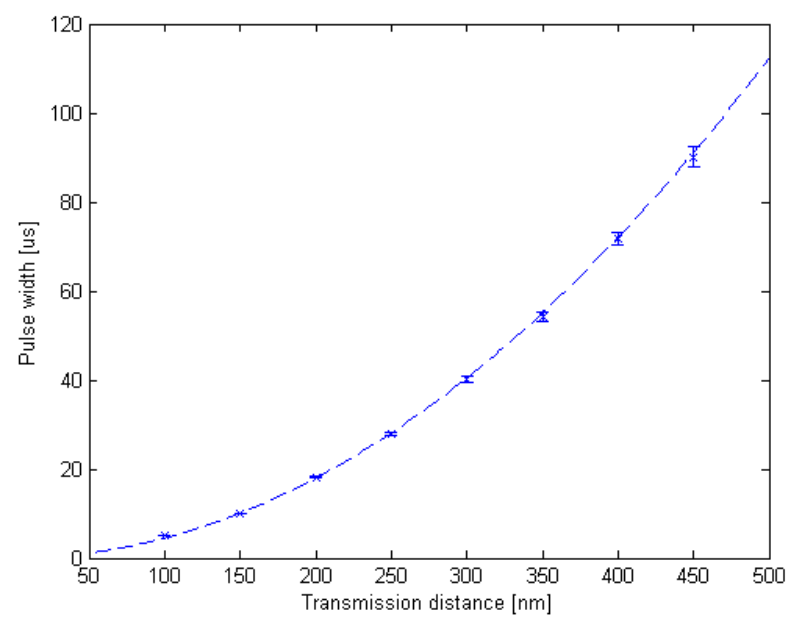

Fig. 8. Plot of the pulse width as a function of the transmission distance. The dashed line corresponds to the analytical expression, and the crosses show the simulation results with $95 \%$ confidence intervals.

In this case, as it happened with the pulse delay, the pulse width is inversely proportional to the diffusion coefficient of the medium. Therefore, the higher the diffusion coefficient, the narrower the received pulses will be.

As before, we validate the obtained expression with N3Sim, using the same parameters as in the previous sections. Fig. 8 shows that the simulation results are close to the values of the analytical expression, which confirms the validity of Eq. (8).

\section{Molecular vs Wireless EM Channel Comparison}

It is worth comparing the communication performance metrics previously found for a diffusion-based molecular communication channel to their equivalent in a wireless electromagnetic (EM) communication channel. In the molecular channel, as we observe in Eq. (3), the pulse delay is proportional to the square of the transmission distance: $t_{d}=\Theta\left(r^{2}\right)$. This is due to the peculiarities of the Brownian motion underlying the diffusion process, which is fundamentally different from the wave propagation observed in EM communications. In the latter case, the propagation delay is equal to the transmission distance divided by the wave propagation speed: $t_{d}=\Theta(r)$.

Eq. (4) shows that the amplitude of a molecular pulse is inversely proportional to the third power of the transmission distance, i.e., $c_{\max }=\Theta\left(1 / r^{3}\right)$. This dependence shows again a difference with respect to the behavior of waves in wireless EM communications, for which, according to the free-space path loss formula $A=\left(\frac{4 \pi r f}{c}\right)^{2}$, the pulse amplitude decreases proportionally to the square of the transmission distance.

Finally, according to Eq. (8), the dependence of the pulse width on the transmission distance is $t_{w}=\Theta\left(r^{2}\right)$. Again, there is a clear difference with the traditional EM channel, for which the pulse width is independent from the transmission distance: $t_{w}=\Theta(1)$. Also, the behavior of the molecular channel differs from that observed in optical communications, where chromatic dispersion causes the pulse width to increase

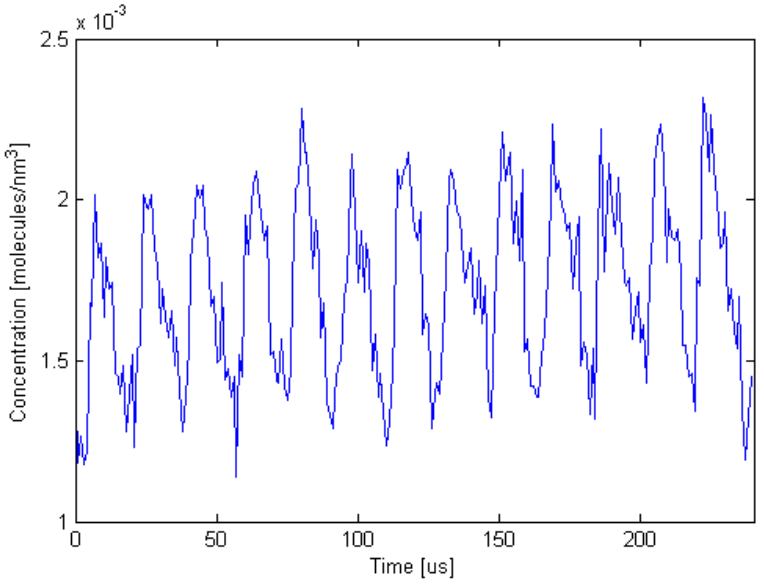

Fig. 9. Concentration measured by the receiver when a train of pulses is transmitted from a distance of $200 \mathrm{~nm}$. The interval between pulses is equal to the pulse width at the receiver location.

proportionally to the transmission distance $\left(t_{w}=\Theta(r)\right)$, at a slower rate than in the molecular channel.

In the following table, we summarize these comparisons between the scalability of communication metrics in the molecular channel and the wireless electromagnetic channel:

\begin{tabular}{l|c|c} 
Metric & EM channel & Molecular channel \\
\hline Pulse delay & $\Theta(r)$ & $\Theta\left(r^{2}\right)$ \\
Pulse amplitude & $\Theta\left(1 / r^{2}\right)$ & $\Theta\left(1 / r^{3}\right)$ \\
Pulse width & $\Theta(1)$ & $\Theta\left(r^{2}\right)$
\end{tabular}

\section{ACHIEVABLE BANDWIDTH}

The pulse width expression, described in Eq. (8), allows us to obtain an estimate on the achievable bandwidth of the pulsebased modulation scheme. Let us consider the case where a nanomachine needs to transmit a bit stream. With this purpose, it may send a train of pulses by using different molecule types to represent bits ' 0 ' and ' 1 ', respectively. In this case, the minimum separation between the transmitted pulses needs to be approximately equal to the pulse width at the receiver, so that pulses can be correctly distinguished and the information can be decoded (assuming that the transmitter and receiver are synchronized). The achievable bandwidth in this scenario is thus approximately equal to the inverse of the pulse width at the receiver location.

The transmitter is able to compute the pulse width at the receiver by using Eq. (8) and it can set the interval between the transmitted pulses accordingly. For example, for a transmission distance of $200 \mathrm{~nm}$, the received pulse width will be of $18 \mu \mathrm{s}$. Fig. 9 represents the received signal when a train of pulses is transmitted by a nanomachine located $200 \mathrm{~nm}$ away with an interval between pulses equal to the pulse width at the receiver $(18 \mu \mathrm{s})$. The simulation results show a stream of distinguishable pulses and thus confirm that the transmitted signal can be correctly decoded by the receiver. 


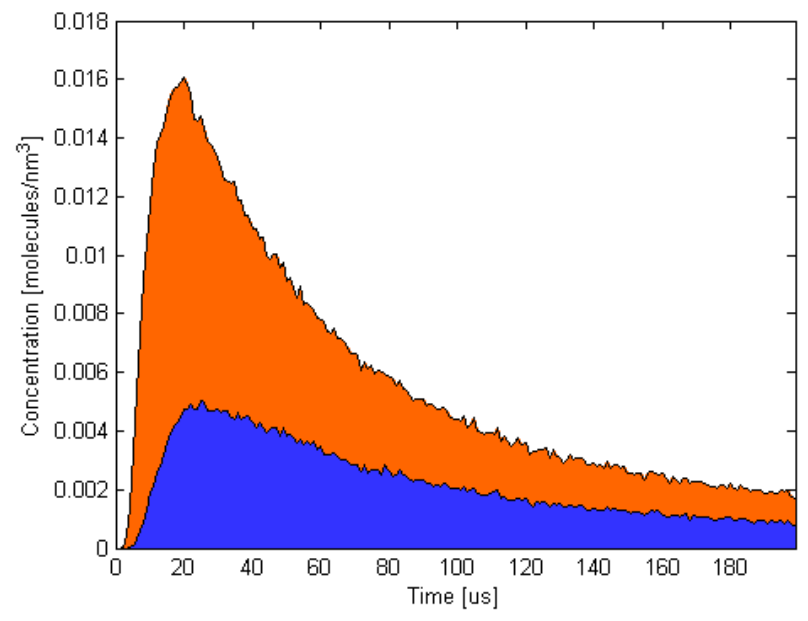

Fig. 10. Concentration measured by the receiver when two transmitters simultaneously emit a molecular pulse. Transmitter 1 (orange) is located at a distance of $300 \mathrm{~nm}$, and transmitter 2 (blue) at $400 \mathrm{~nm}$.

\section{Multiple TransmitTers}

In order to demonstrate the feasibility of a scenario where multiple nanomachines are transmitting simultaneously, we perform a simulation where two transmitters emit a pulse of molecules at the same time instant. Fig. 10 shows the concentration measured by a receiver in this scenario. The transmitter nanomachines are located at a distance of 300 and $400 \mathrm{~nm}$, respectively, and they use different molecule types in order to avoid the collision of the transmitted pulses. Since the transmitted molecules do not interact, the pulses are orthogonal and they can be successfully decoded by the receiver.

\section{CONCLUSION}

In this paper, we have characterized the diffusion-based communication channel in molecular nanonetworks. First, we have described our envisaged scenario, where molecules emitted by transmitter nanomachines diffuse throughout the medium according to Fick's laws of diffusion. We have then obtained the impulse response, the transfer function and the group delay of the molecular channel.

We have proposed a pulse-based modulation scheme which we consider specially well suited to the molecular environment. Based on this scheme, we have obtained analytical expressions for relevant metrics from the communication standpoint: pulse delay, pulse amplitude and pulse width. We have also validated these results by means of simulations and showed the difference in the scalability of the obtained metrics with respect to their equivalent in wireless electromagnetic communication. Finally, we have explored the achievable bandwidth by the proposed communication system and we have demonstrated the feasibility of a scenario with multiple simultaneous transmissions.
We consider that these results provide interesting insights which may serve designers as a guide to implement future molecular nanonetworks.

\section{ACKNOWLEDGMENT}

This work has been partially supported by the FPU grant of the Spanish Ministry of Education.

\section{REFERENCES}

[1] R. A. Freitas, "Nanotechnology, nanomedicine and nanosurgery." International Journal of Surgery, vol. 3, no. 4, pp. 243-6, Jan. 2005.

[2] C. M. J. Pieterse and M. Dicke, "Plant interactions with microbes and insects: from molecular mechanisms to ecology," Trends in plant science, vol. 12, no. 12, pp. 564-9, Dec. 2007.

[3] J. Han, J. Fu, and R. B. Schoch, "Molecular sieving using nanofilters: past, present and future." Lab on a chip, vol. 8, no. 1, pp. 23-33, Jan. 2008.

[4] I. F. Akyildiz, F. Brunetti, and C. Blázquez, "Nanonetworks: A new communication paradigm," Computer Networks, vol. 52, no. 12, pp. 2260-2279, 2008.

[5] J.-Q. Liu, "Molecular informatics of nano-communication based on cells: A brief survey," Nano Communication Networks, vol. 1, no. 2, pp. 118-125, Jun. 2010.

[6] E. Carafoli, "Calcium signaling: a tale for all seasons." Proc. of the National Academy of Sciences of the United States of America, vol. 99 , no. 3, pp. 1115-22, Feb. 2002.

[7] T. Nakano, T. Suda, M. J. Moore, R. Egashira, A. Enomoto, and K. Arima, "Molecular communication for nanomachines using intercellular calcium signaling," in Proc. IEEE Conference on Nanotechnology. IEEE, 2005, pp. 632-635.

[8] F. Walsh, S. Balasubramaniam, D. Botvich, and W. Donnelly, "Review of communication mechanisms for biological Nano and MEMS devices," in 2nd Bio-Inspired Models of Network, Information and Computing Systems. IEEE, Dec. 2007, pp. 307-312.

[9] M. Pierobon and I. F. Akyildiz, "A physical end-to-end model for molecular communication in nanonetworks," IEEE Journal on Selected Areas in Communications, vol. 28, no. 4, pp. 602-611, 2010.

[10] B. Atakan and O. Akan, "On molecular multiple-access, broadcast, and relay channels in nanonetworks," in International Conference on BioInspired Models of Network, Information and Computing Sytems. ICST (Institute for Computer Sciences, Social-Informatics and Telecommunications Engineering), 2008.

[11] M. Pierobon and I. F. Akyildiz, "Information Capacity of Diffusionbased Molecular Communication in Nanonetworks," in Proc. IEEE Infocom Miniconference, 2011.

[12] D. Arifler, "Capacity Analysis of a Diffusion-Based Short-Range Molecular Nano-Communication Channel," Computer Networks, Dec. 2010.

[13] D. E. Clapham, "Calcium signaling," Cell, vol. 131, no. 6, pp. 1047-58, Dec. 2007.

[14] J. Philibert, "One and a Half Century of Diffusion: Fick, Einstein, before and beyond," Diffusion Fundamentals, vol. 4, no. 6, pp. 1-19, 2006

[15] T. S. Ursell, "The Diffusion Equation A Multi-dimensional Tutorial," Pasadena, 2007.

[16] H.-Y. Yeh, M. V. Yates, W. Chen, and A. Mulchandani, "Real-time molecular methods to detect infectious viruses." Seminars in cell \& developmental biology, vol. 20, no. 1, pp. 49-54, Feb. 2009.

[17] W. H. Bossert and E. O. Wilson, "The analysis of olfactory communication among animals." Journal of theoretical biology, vol. 5, no. 3, pp. 443-69, Nov. 1963.

[18] L. Parcerisa and I. F. Akyildiz, "Molecular communication options for long range nanonetworks," Computer Networks, vol. 53, no. 16, pp. 2753-2766, 2009.

[19] "NaNoNetworking Center in Catalunya," http://www.n3cat.upc.edu/ n3sim.

[20] B. S. Donahue and R. Abercrombie, "Free diffusion coefficient of ionic calcium in cytoplasm," Cell Calcium, vol. 8, no. 6, pp. 437-448, 1987.

[21] R. M. Corless, G. H. Gonnet, D. E. G. Hare, D. J. Jeffrey, and D. E. Knuth, "On the Lambert W function," Advances in Computational Mathematics, vol. 5, no. 1, pp. 329-359, Dec. 1996. 